\title{
Laboratory studies of the dynamic properties of soils of weak bases using the Fourier method
}

\author{
Sergey Kudryavtsev ${ }^{1 *}$ and Vladlen Stefanyuk ${ }^{1}$ \\ ${ }^{1}$ Far Eastern State Transport University, 47, Seryshev str., Khabarovsk, 680021, Russia
}

\begin{abstract}
The article discusses the cyclic triaxial compression method and the resonance column method for determining the dynamic properties of soils, as well as the Fourier method used to evaluate the spectral composition.
\end{abstract}

\section{Introduction}

During building high embankments on a weak foundation in seismically hazardous areas, there is a need to magnifying the bearing capacity of soils. In the Far Eastern region of Russia, located on the coast of the Sea of Okhotsk, seismicity from 7 to 9 points is observed. For understood highlights conduct materials within the conditions of seismic activity, to know the composition of the soil, the apparent amount of its constituent materials , the nature and conditions of the interaction of all phases determining the materials properties. In forecast a materials behavior, it is to establish the physical characteristics of the materials, which is meant to be used on the basis of dynamic calculation ah structures.

To find dynamically or seismic reaction and materials when designing and it is necessary to determine the dynamic properties of materials by Lab and field tests in conditions of dynamic loading.

The process is described two method and test materials under conditions of dynamic effects : 1) the cyclic triaxial compression, which is used for determining the dynamic resistance of saturated materials by cyclic triaxial controlled load and shear modulus and damping coefficient ; 2) low-amplitude dynamic vibrations (resonance column) to determine the shear wave velocity, shear modulus and materials damping coefficient in a small range of shear deformations.

In laboratory tests mean the cyclic triaxial compression method, data are obtained with a high degree of accuracy for high strain levels, the results of which can be used in the integral Fourier transform. It has a number of properties that are convenient to use in solving dynamic problems and for describing seismic effects. Fourier spectra are widely used in engineering seismology and serve as the main means of assessing the spectral composition of recorded ground movement or structure reactions [1-14].

*olgakudr56@mail.ru 


\section{The device for cyclic triaxial compression}

The dynamic properties of materials are characterized by three parameters: elastic or initial shear modulus $\mathrm{G}$, damping coefficient $\mathrm{D}$ and shear wave velocity Vs, measurements of $\mathrm{D}$ and $\mathrm{G}$ are carried out independently, both in the field and in the laboratory. Laboratory more accurate. [1]

Many other factors can affect the dynamic properties of the materials. For example, consolidation pressure, porosity coefficient, geological age, cementation, stress history, ductility number, strain rate and number of cycles can have a significant effect on D, G and linear dynamics of properties. [2,4]

The cyclic triaxial compression method is used to determine the dynamic strength (dilution potential) of water-saturated materials and the shear modulus and damping coefficient. Water-saturated loose materials subjected to non-drained cyclic loading can lose their strength and undergo deformation due to liquefaction. The main reason for liquefaction is the development of excess steam pressure in the absence of drainage, which leads to a decrease in effective stresses between materials particles. The stiffness is reduced and thus the materials becomes less durable. At zero effective stresses, sand particles "float" in pore water with minimal contacts between each other, and the soil loses its shear resistance and stiffness passes into a fluid state. There are several types of dilution: flow dilution, cyclic softening, cyclic resistance, cyclic dilution. [2]

Structurally, the installation is made in the form of a frame with a rigid traverse and racks. On the baseplate of the frame is set transparent cylindrical Skye chamber plexiglass rings of carbon fiber reinforced. The bottom plate of the working chamber has connectors for connecting sensors. The sample is placed inside the working chamber. Lateral and back pressure on the sample is created using a pressure control system. On located crosspiece electromechanical actuator, he create static and kinematic axial load, including in the dynamic mode at a predetermined frequency and amplitude on the generators sample was through special active stamp.

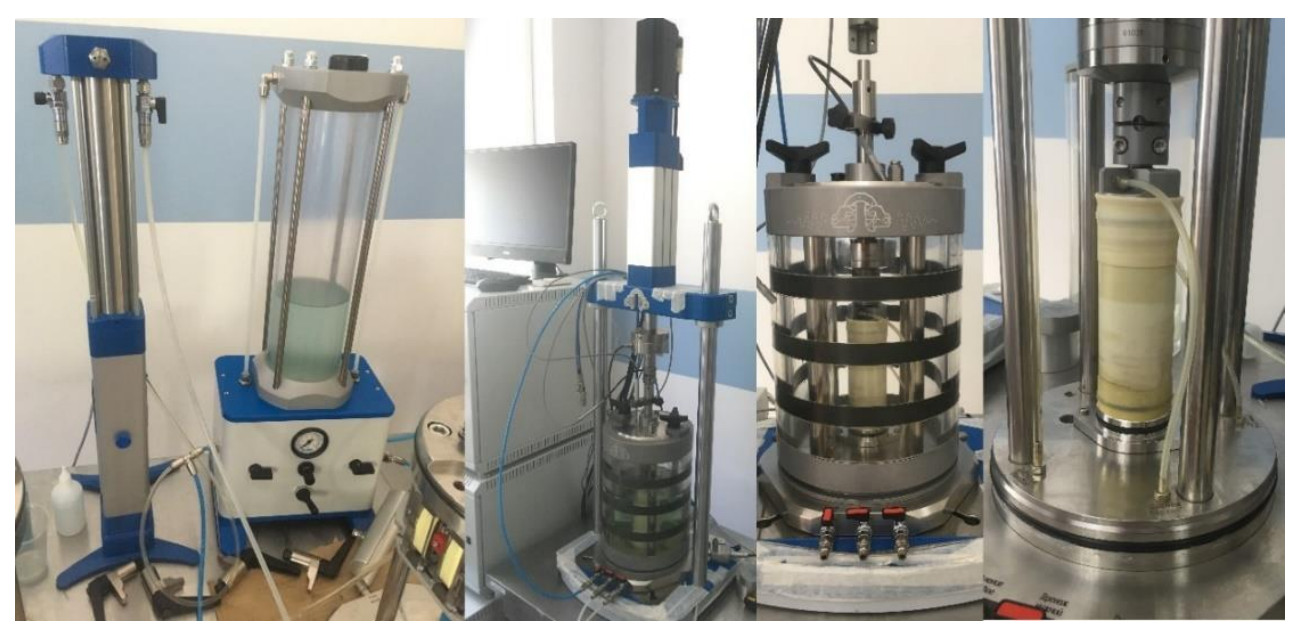

Fig. 1 Cyclic triaxial compression device. 
During testing of the measuring system controls axial load and axial deformation of the sample, and the opposite side neutral pressures, the volumetric deformation of the specimen. Cyclic triaxial test conducted by the test of continuous cylindrical soil samples at their full loading them pressure (isotropic compression) by applying axial and cyclic load capabilities without drainage. $[2,5,6]$

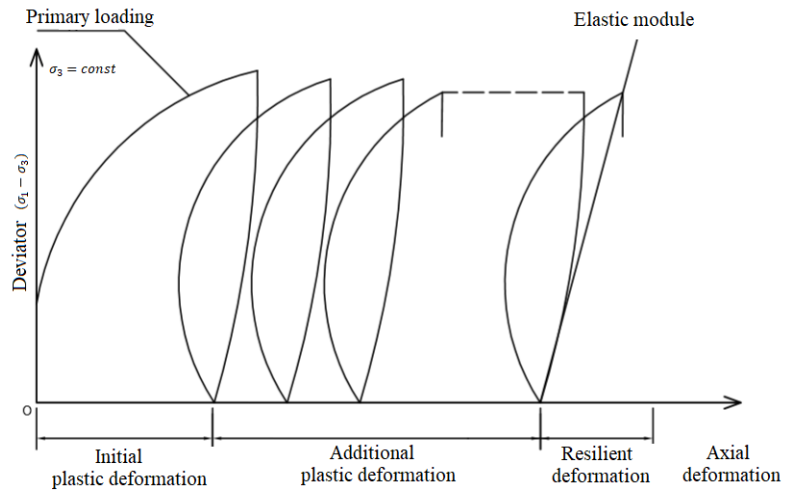

Fig. 2 Typical deformation behavior of soil under cyclic loading dependence of axial deformation on the deviator in tests with load control.

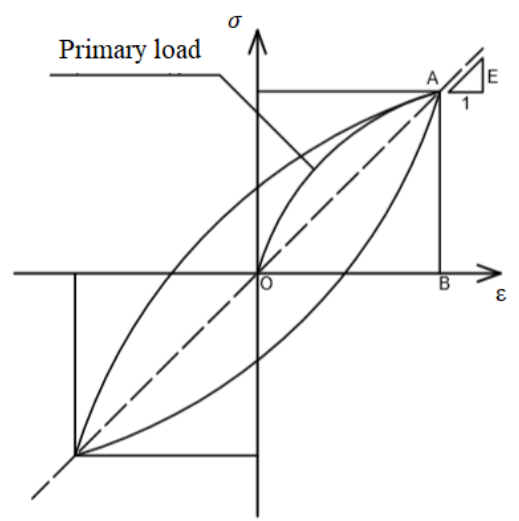

Fig. 3 Typical deformation behavior of soil under cyclic loading theoretical curve of one loading and unloading cycle

Test work are carried out in three stages: water saturation, consolidation and cyclic loading. After the samples are completely water saturated, in the second stage, they undergo isotropic or anisotropic consolidation. When consolidation is complete, soil samples are loaded sinus sinusoidal varying load. [5]

During cyclic triaxial with isotropic or anisotropic consolidated soil samples voltage act on a plane inclined at an angle of $45^{\circ}$ to the horizontal, and model the stresses acting on the horizontal plane of the ground element in the field under seismic loading. On this plane applied on Booting creates a shearing stress equal to half the voltage deviator subjecting to stress and the normal stress equal to the average voltage; tangent voltage of $[2,5]$

By changing the lateral pressure in the working chamber of the triaxial device with the application of an axial voltage deviator, a standard cyclic triaxial test can be carried out so that the average total voltage remains constant, as occurs during an earthquake. 
The researchers took two different criteria to determine the soil liquefaction. First, the number of cycles at which the excess pore pressure is camping to an initial effective hydrostatic pressure. The second determines the onset of liquefaction based on axial strain; the sample is diluted when the axial strain to attains its $5 \%$ double strain amplitude $( \pm 2,5 \%$ axial strain). [3]

In experiments to assess the capacity of soil to liquefaction amplitude di -dynamic load is set at a corresponding coefficient cyclic subjecting to stress $C S R$, which is defined as the ratio of the maxi -formal applied shear stress to the initial average subjecting to stress (the pressure in the working chamber of the device). $[2,7]$

$$
\operatorname{CSR}=\frac{\tau}{\sigma_{3}}=\frac{1}{2} \frac{\left(\sigma_{1}-\sigma_{3}\right)}{\sigma_{3}}=\frac{\sigma_{\mathrm{d}}}{2 \sigma_{3}}
$$

where $\tau$ - is the maximum tangential stress; $\sigma_{3}$ - comprehensive pressure at the end of the consolidation phase; $\sigma_{1}-\sigma_{3}-$ subjecting to stress deviator acting during cyclic loading. For analysis of potential soil to liquefaction be performed from three to five trials with different values of cyclic Skog voltage identically prepared soil samples. [7]

The parameters of loose and cohesive soils determined by this method depend on many factors, including the level of deformation, density, number of cycles, soil type and effective stresses.

In general, the storage modulus $E_{s e c}$ can be calculated from the cyclic triaxial angle of inclination of the line passing through the vertex of the hysteresis loop, depending on "the subjecting to stress axial of the axial strain," and is meant to be used expression:

$$
\mathrm{E}=\frac{\Delta \sigma_{\mathrm{d}}}{\Delta \varepsilon}=\frac{\left(\sigma_{\mathrm{d}, \max }-\sigma_{\mathrm{d}, \min }\right)}{\left(\varepsilon_{\max }-\varepsilon_{\min }\right)} ; \mathrm{D}=\frac{\mathrm{A}_{\mathrm{L}}}{4 \pi \mathrm{A}_{\Delta}}
$$

where $\Delta \sigma_{d}$ - is the amplitude of the voltage deviator; - the amplitude of axial deformation. [2, 17]

At the same time, using the test data by plotting deformation shear stress find the shear modulus:

$$
\mathrm{G}=\frac{\tau_{\mathrm{DA}}}{\gamma_{\mathrm{DA}}} ; \mathrm{D}=\frac{\mathrm{A}_{\mathrm{loop}}}{4 \pi \mathrm{A}_{\Delta}}
$$

\section{Resonance column method}

The resonant column method is used to determine shear wave velocity, shear modulus, and materials damping coefficient in the range of shear deformation from $10^{-6}$ to $10^{-3}$. Resonant columns provide more consistent test results and tests themselves are considered one of the most accurate methods for determining the dynamic properties of soils at low shear $\left(10^{-3}<\mathrm{y}<10^{-6}\right) .[2]$

Torsional vibrations are created by four magnetoelectric drives all together it is a torsional drive. The magnetoelectric drive consists of an inductor and a core a permanent magnet. The angular displacement measurement system consists of an accelerometer and several noncontact sensors. Generated torque is about proportional to the current flowing in the coils, which are not controlled indirectly via precision electronic amplifier. Systems ma control and data acquisition controls all measurement channels. [7] 


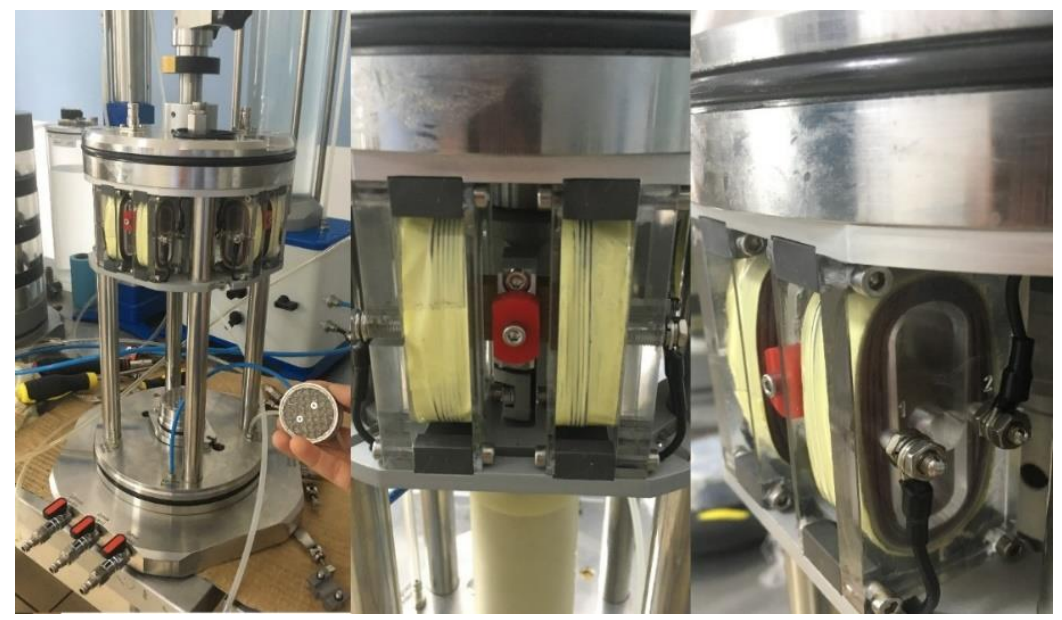

Fig. 4 Resonance column

Vertical deformation is measured by a displacement sensor. Greatness to shear deformation is controlled by the angle of twist for samples of solid section. Vibration displacement is measured by accelerometers. After sample preparation and isotropic soil consolidation be subjected torque strength with claim amplitude variable and often one. The loading range should include the induced resonant frequency. [2, 7]

The software must control equipment, col host data, and then process the information, which, together with the parameter $\mathrm{E}$ and the calibration information of samples computes dynamic properties of soils. [2]

Data analysis Standard Test Method resonant columns assumes elastic behavior of the system with zero damping in determining the shear modulus. The damping factor is calculated independently of the shear modulus, using a free vibration method polo fault bandwidth transfer function or method.

In the standard model base sample column resonance thawed fixed and the torsional load is created in the upper part of the sample. The bottom of the sample is mounted on a fixed plate, and the top is adjacent to the active plate. The active plate has a rotational inertia mass $\mathrm{J}_{\mathrm{A}}$ and partly limited by a spring with stiffness $\mathrm{K}_{\mathrm{A}}$ and a damper constant $\mathrm{J}_{\mathrm{A}}, \mathrm{K}_{\mathrm{A}}, \xi_{\mathrm{A}}$. Dynamic properties of the active plate $J_{A}, K_{A}, \xi_{A}$. known from calibration tests. $[5,6,7,8]$

Equation ( 4 ) is used to calculate the shear wave velocity of a material:

$$
\mathrm{V}_{\mathrm{s}}=\frac{2 \pi * \mathrm{f}_{\mathrm{r}} * \mathrm{H}}{\beta}
$$

where $\beta$ - calculated from the polar moment of inertia of the mass of the sample and the polar angular momentum of inertia of the actuator plate; $f_{r}$ - first measured shape Lakes resonance frequency, $\mathrm{Hz} ; H$ - height of the sample.

The shear modulus can be calculated as:

$$
\mathrm{G}=\mathrm{pV}_{\mathrm{s}}^{2}
$$

The damping coefficient of the sample material is calculated by vibration damping or by half bandwidth.

1. The method of free damping of oscillations. Here uses a smiling solution system with one degree of freedom with viscous damping proposed. The process of free vibration damping of a system with one degree of freedom is described by the decrement of the logarithm, which is the ratio of the natural logarithm of two successive amplitudes of motion:

$$
\mathrm{u}=\ln \left(\frac{\mathrm{Z}_{1}}{\mathrm{Z}_{2}}\right)=\frac{2 \mathrm{nD}}{\sqrt{1-\mathrm{D}^{2}}}
$$

where $Z_{1}$ and $Z_{2}$ - two consecutive amplitudes of motion; $D$ is the damping coefficient of 
the material; $n$ is the number of cycles. [2]

2. Half bandwidth method. Based on the measurement wideness us frequency near the resonance curve (Fig. 10). From the curve of the frequency of click can calculate the logarithmic decrement, using the expression:

$$
\mathrm{u}=\frac{\mathrm{n}}{2} \frac{\mathrm{f}_{2}-\mathrm{f}_{1}}{\mathrm{f}_{\mathrm{r}}^{2}} \sqrt{\frac{\mathrm{A}^{2}}{\mathrm{~A}_{\text {max }}^{2}-\mathrm{A}^{2}}} \frac{\sqrt{1}-2 \mathrm{D}^{2}}{1-\mathrm{D}^{2}}
$$

where $f_{1}$ - is the frequency below resonance, where is the strain amplitude, $A ; f_{2}$ - is the frequency above resonance, where the strain amplitude is the resonance frequency; $D$ - is the damping coefficient; $n$ - is the number of cycles.[2]

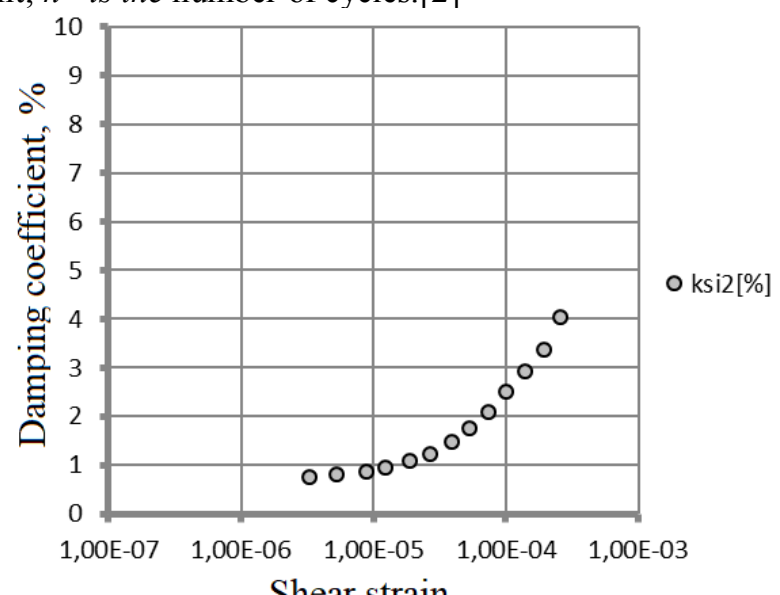

Shear strain

Fig. 5 Damping / warping

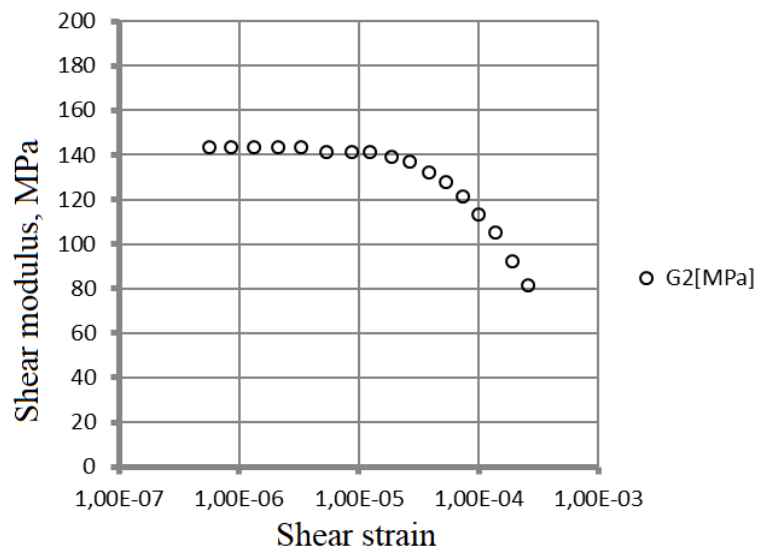

Fig. 6 Shear / deformation modulus 


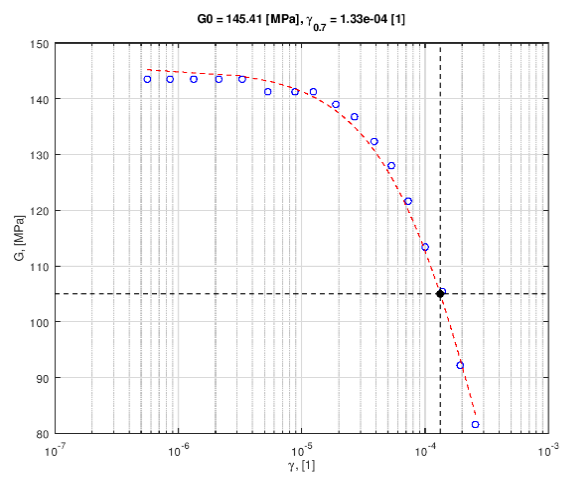

Fig. 7 Schedule Shear Modules, for Plaxis

Based on the results of processing the protocol on the resonant column, graphs are plotted. A graph of the damping coefficient according to the regime of free damped oscillations calculated from the values obtained during the test is constructed. It was possible to calculate damping coefficients for free vibrations only for strains starting from 3.29E-06. The results of approximation of the shear modulus graph for solving geotechnical problems in various programs are also presented . $[1,4,8]$

\section{Analysis of the response spectrum}

The response spectrum is a graph of maximum reactions: displacements, speeds, accelerations for a given impact. One of the main tools for seismic design at present. It is defined as summing the response peaks of the entire possible linear-elastic system with one degree of freedom. For different damping values, there will be different forms of response spectra. $[14,15]$

To take into account the damping properties of various building materials, it is necessary to build a family of spectral curves with different damping coefficients. In this case, laboratory tests were performed where the damping value was determined. But in normative documents it is recommended to use the established damping factors for various materials.

Fourier spectra are widely used in engineering seismology and serve as the main means for assessing the spectral composition of recorded ground movement or the reaction of structures, but other functions are determined using Fourier spectra, such as power spectral density functions, transfer functions, and coherence functions. [11]

To construct the response spectra, it is necessary to solve the differential equation:

$$
\ddot{u}_{r}+2 \xi \omega \dot{u}_{r}+\omega^{2} u_{r}=-\ddot{u}_{g}(t)
$$

which describes the relative vibrations of a system with one degree of freedom for a given vertical or horizontal vibration of the base [12]

Consider the oscillations of the soil when exposed to several earthquakes. Peak duration of grounding acceleration and frequent earthquake content can be obtained from accelerograms. 


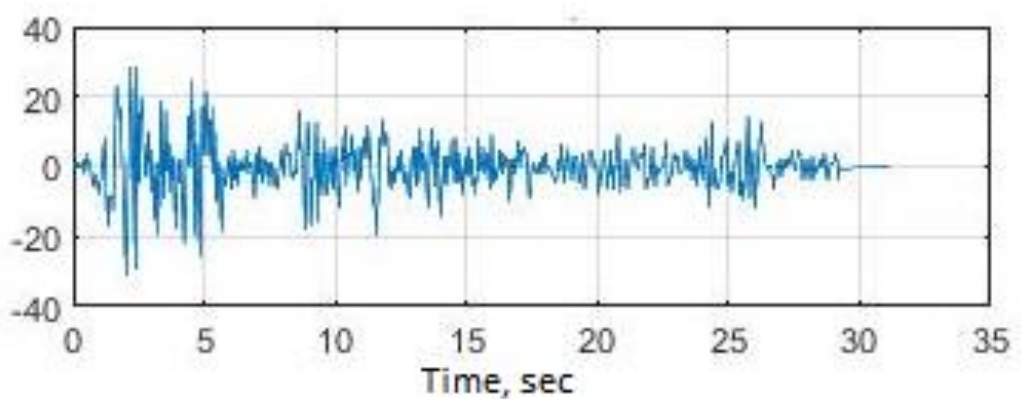

Fig. 8 Accelerogram of ground surface vibrations in California

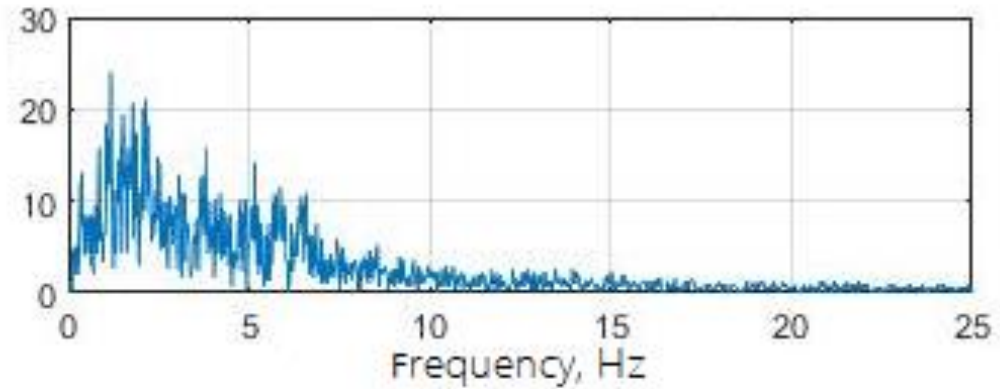

Fig. 9 Amplitude spectrum

The amplitude spectrum of accelerations is determined by the Fourier transform:

$$
\tilde{\mathrm{u}}_{\mathrm{g}}(\omega)=\mathrm{F}\left[\ddot{\mathrm{u}}_{\mathrm{g}}(\mathrm{t})\right]=\int_{0}^{\mathrm{t}_{\text {кон }}} \ddot{\mathrm{u}}_{\mathrm{g}}(\mathrm{t}) \mathrm{e}^{\mathrm{i} \omega \mathrm{t}} \mathrm{dt}
$$

Under seismic influences, the soil vibration spectra do not contain harmonics with frequencies exceeding 25-30 Hz. Under anthropogenic impacts, the spectrum of vibrations can be wider and contain harmonics with higher frequencies. For example, vibrations propagating during the movement of railway trains contain harmonics up to $100 \mathrm{~Hz}$. [ 9, $10,11]$

The spectra of maximum velocities and spectra of maximum accelerations can be obtained in the same way as the spectra of maximum displacements were determined. The difference will be that instead of defining peak displacement values, it will be necessary to determine peak velocity values and peak acceleration values. Instead, an approximate method is used, assuming that the time dependence of the displacement spectrum is a harmonic function. In this case, the spectrum of maximum velocities is determined by the expression, which follows from the rule of differentiation of harmonic functions. Since the velocity spectrum thus obtained is not accurate, it is called the pseudospectrum. [11, 14]

It can be shown that to construct the spectra, it is sufficient to determine only the spectrum of pseudo-velocities using the formula:

$$
S_{p v}(\omega, \xi)=\left[\int_{0}^{t} \ddot{u}_{g}(\tau) \sin \omega(t-\tau) e^{-\omega \xi(t-\tau)} d \tau\right]_{\max }
$$

where the index " max" means the maximum value for the function module for the entire time interval.

$$
\begin{gathered}
S_{d}(\omega, \xi)=\frac{S_{p v}(\omega, \xi)}{\omega} \\
S_{v}(\omega, 0)=\left[\int_{0}^{t} \ddot{u}_{g}(\tau) \cos \omega(t-\tau) d \tau\right]_{\max }
\end{gathered}
$$




$$
\mathrm{S}_{\mathrm{pv}}(\omega, 0)=\left[\int_{0}^{\mathrm{t}} \ddot{\mathrm{u}}_{\mathrm{g}}(\tau) \sin \omega(\mathrm{t}-\tau) \mathrm{d} \tau\right]_{\max }
$$

Both expressions are identical, with the exception of trigonometric functions. Since the expression differs from the real spectrum, it is commonly called the pseudo- spectrum .

When $\xi=0$ it follows:

$$
S_{a}(\omega, 0)=\left[\omega \int_{0}^{t} \ddot{u}_{g}(\tau) \sin \omega(t-\tau) d \tau\right]_{\max }
$$

thus follows:

$$
\mathrm{S}_{\mathrm{a}}(\omega, 0)=\omega \mathrm{S}_{\mathrm{pv}}(\omega, 0)
$$

With a damping coefficient defined by the range $0<\xi<0.2$, you can use the approximate ratio:

$$
\mathrm{S}_{\mathrm{a}}(\omega, \xi) \approx \omega \mathrm{S}_{\mathrm{pv}}(\omega, \xi)
$$

The pseudo-acceleration spectrum is the absolute acceleration of the system. $\mathrm{P}$ acceleration is determined by the expression:

$$
\mathrm{S}_{\mathrm{pa}}(\omega, \xi)=\omega \mathrm{S}_{\mathrm{pv}}(\omega, \xi)
$$

For systems with low damping coefficients, which include, in practice, most building structures, the following relations are true:

$$
S_{p v}(\omega, \xi)=\omega S_{d}(\omega, \xi)=\frac{S_{p a}(\omega, \xi)}{\omega}
$$

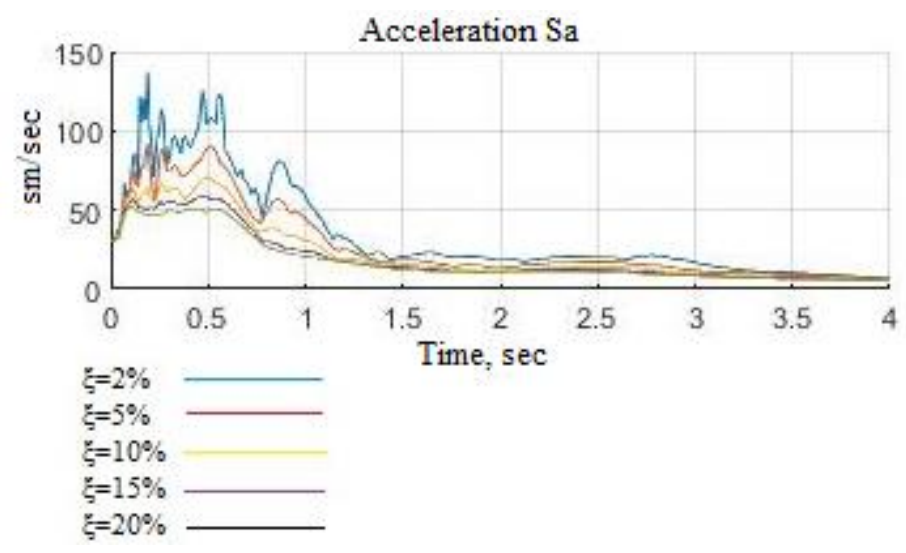

Fig. 10 Range of maximum responses in accelerations (El Centro) 


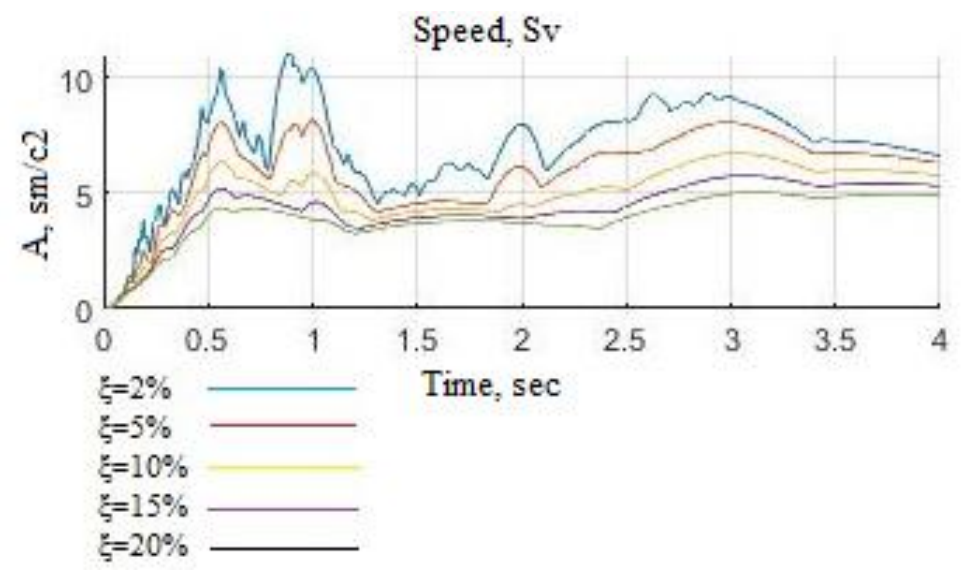

Fig. 11. Range of maximum responses in speeds (El Centro)

A comparison between the spectra for different accelerograms leads to the following:

- in a system without damping, the results are almost the same for a period of less than one second $(\mathrm{T}<1 \mathrm{~s})$;

- with increasing damping to $20 \%$, the differences are within $20 \%$ but without any noticeable bias;

- the pseudo-acceleration spectrum is more accurate than the pseudo-speed spectrum. [11, $12,17]$

\section{Conclusion}

For the design of buildings and structures subject to seismic and dynamic loads, for successful analysis, it is important to measure the dynamic properties of the soil. A reliable assessment of the dynamic properties of the soil can only be made using various laboratory test methods, which are characterized by shear modulus, damping coefficient and cyclic strength. These characteristics are the most important properties that determine the behavior of soils under seismic and dynamic loads.

Using the results of laboratory tests, applying them to accelerograms of vibrations of the soil surface of a real earthquake, we can build graphs of the maximum reactions (response spectrum). Namely, taking into account the damping properties. Using different laboratory methods, having obtained a damping coefficient, a spectrum of responses with different periods of natural oscillations is constructed.

Laboratory tests in conjunction with spectral analysis and taking into account all the dynamic characteristics of the soil will give a more accurate solution to the problem than using the "dynamic coefficients" used in the norms of the Russian Federation when calculating seismic resistance. The spectrum can be represented as in norms in a standard form, but can be calculated according to an individual plan, because not all construction sites are the same.

The spectrum of responses to earthquakes has a clear and understandable physical meaning, it is well covered in foreign scientific sources, in contrast to the "dynamic coefficient" for which there is no definition and how to calculate it. 


\section{References}

1. B. Kovačič, R. Kamnik, A. Pustovgar, N. Vatin. Procedia Engineering. 2016. 165. Pp. 918-925. DOI:10.1016/j.proeng.2016.11.801.

2. M. Lazarevska, M. Cvetkovska, M. Knezevic, A. Trombeva Gavriloska, M. Milanovic, V. Murgul, N. Vatin. Applied Mechanics and Materials. 2014. 627. Pp. 276-282. DOI:10.4028/www.scientific.net/AMM.627.276.

3. B. Melović, S. Mitrović, A. Djokaj, N. Vatin. Procedia Engineering. 2015. 117(1). Pp. 802-807. DOI:10.1016/j.proeng.2015.08.255.

4. V. Murgul, D. Vuksanovic, N. Vatin, V. Pukhkal. Applied Mechanics and Materials. 2014. 680. Pp. 524-528. DOI:10.4028/www.scientific.net/AMM.680.524.

5. N. Vatin, N. Lavrov, G. Loginov. MATEC Web of Conferences. 2016. 73. DOI:10.1051/matecconf/20167301006.

6. N. Vatin, A. Sinelnikov, M. Garifullin, D. Trubina. Applied Mechanics and Materials. 2014. 633-634. Pp. 1037-1041. DOI:10.4028/www.scientific.net/AMM.633634.1037

7. L. Lazarević, D. Vučković, Z. Popović, Journal of Rail and Rapid Transit 230(8), 18281841 (2016).

8. M. Vilotijević, M. Vukićević, L. Lazarević, Z. Popović,Tehnički vjesnik 25(2), 516523 (2018).

9. L. Lazarević, D. Vučković, M. Vilotijević, Z. Popović, Structural Health Monitoring 18(3), 792-805 (2019).

10. V. Vorobyov, A. Manakov, I. Yanshina, I. Repina, Advances in Intelligent Systems and Computing, VIII International Scientific Siberian Transport Forum 2, 1116 (2020). DOI: 10.1007/978-3-030-37919-3_69

11. A.L. Manakov, A.D. Abramov, A.S. Ilinykh, M.S. Galay, J.S. Sidorov, Journal of Physics: Conference Series 1050(1), 012051 (2018). DOI: 10.1088/17426596/1050/1/012051

12. K. Kornienko, S. Bessonenko, I. Tanaino, Advances in Intelligent Systems and Computing 1115, 655-662 (2020). DOI: 10.1007/978-3-030-37916-2_63

13. Tanaino, O. Yugrina, L. Zharikova, IOP Conference Series: Earth and Environmental Science 403(1), 012192 (2019). DOI: 10.1088/1755-1315/403/1/012192

14. S. Bessonenko, K. Kornienko, I. Tanaino, MATEC Web of Conferences 239, 03002 (2018). DOI: $10.1051 /$ matecconf $/ 201823903002$ 